\title{
Increasing Intestinal Soy Protein Hydrolysate Levels Promotes Colon Inflammation By Affecting Microbial Metabolism and Increases the Protein Expression of Colonic Tight Junction Proteins
}

\section{Zhongxin Li}

Laboratory of Gastrointestinal Microbiology, Nanjing Agricultural University

\section{Liren Ding}

National Experimental Teaching Center for Animal Science, College of Animal Science and Technology,

Nanjing Agricultural University

\section{Weiyun Zhu}

Laboratory of Gastrointestinal Microbiology, Nanjing Agricultural University

Suqin Hang ( $\nabla$ suqinhang69@njau.edu.cn )

Laboratory of Gastrointestinal Microbiology, Nanjing Agricultural University

\section{Research Article}

Keywords: Soy protein hydrolysate, growing pig, colonic microbiota, metabolic profile, inflammatory cytokines, tight junction proteins

Posted Date: December 1st, 2021

DOI: https://doi.org/10.21203/rs.3.rs-1095654/v1

License: (c) (i) This work is licensed under a Creative Commons Attribution 4.0 International License.

Read Full License 


\section{Abstract \\ Background}

Increasing intestinal protein levels change the colonic microbial community and metabolic profile of pigs, but the effect of this on colonic inflammation and barrier function in growing pigs remains unclear.

\section{Results}

Sixteen pigs $(35.2 \pm 0.3 \mathrm{~kg}$ ) were infused with sterile saline (control) or soy protein hydrolysate (SPH) (70 $\mathrm{g} /$ day) through a duodenal fistula twice daily during a 15-day experimental period. The SPH treatment did not affect their average daily feed intake and daily weight gain $(P>0.05)$, but reduced colon index and length $(P<0.05)$. Illumina MiSeq sequencing revealed that species richness was increased following SPH intervention $(P<0.05)$. Furthermore, SPH reduced the abundance of butyrate- and propionate-producing bacteria-such as Lachnospiraceae NK4A136 group, Lachnospiraceae_uncultured, Coprococcus 3 Lachnospiraceae UCG-002, and Anaerovibrio-and increased the abundance of potentially pathogenic bacteria and protein-fermenting bacteria, such as Escherichia-Shigella, Dialister, Veillonella, Prevotella, Candidatus Saccharimonas, Erysipelotrichaceae UCG-006, Prevotellaceae_uncultured, and Prevotellaceae UCG-003 $(P<0.05)$. In addition, a lower content of total short-chain fatty acids, propionate, and butyrate and a higher concentration of cadaverine, putrescine, total biogenic amines, ammonia, and isovalerate were observed following SPH infusion $(P<0.05)$. Further analysis revealed that SPH increased the concentration of tumour necrosis factor- $\alpha$, interleukin (IL)-1 $\beta, \mathrm{IL}-6$, and IL-8 in the colonic mucosa $(P<$ 0.05). Interestingly, SPH intervention increased the expression of occluding, zonula occludens (ZO)-1, and claudin-1 in colonic mucosa $(P<0.05)$. Correlation analysis showed that different genera were significantly related to the production of metabolites and the concentrations of pro-inflammatory cytokines.

\section{Conclusion}

Overall, increasing intestinal soy protein levels altered the colonic microbial composition and metabolic profile, which may lead to colon inflammation and may also strengthen the colon barrier function.

\section{Introduction}

Gut health has substantial far-reaching impact on the overall good health of pigs and is affected by numerous factors, such as nutrition, environment, and genetics. Among these, nutritional factors (protein, fat, and carbohydrate levels) play a major role in a pig's gut health[1]. Protein is an indispensable nutrient for the growth of pigs and increasing dietary protein levels can improve a pig's growth performance[2]. However, protein metabolism is generally considered detrimental to the host colon, because the distal colon-primarily where bowel disease begins-is also the main site of protein fermentation[3]. 
Excess dietary protein along with peptides and free amino acids that escape absorption in the small intestine enter the large intestine and are mainly fermented by abundant microbes colonized in the colon[4]. In a rat experiment, high-protein diets (HPD) promoted protein fermentation in the rat's colon and the production of protein metabolites was found to be significantly positively correlated with colon inflammation and barrier function damage[5]. Another study revealed that increasing colonic protein levels increased the risk of diseases (like colon inflammation) in healthy people and potential pathogens in human faecal samples and reduced the abundance of total bacteria and beneficial bacteria (like the butyrate-producing Roseburia/Eubacterium rectale group)[6]. Therefore, colonic microbial disorders and changes in metabolic patterns may be the main reasons for the occurrence of colon diseases due to increased protein levels. Previous studies reported that increased intestinal protein levels altered the composition of colon microbiota in piglets $[7,8]$. The colonic microbiome of pigs can ferment protein in vitro to produce various protein metabolites, such as biogenic amines and branched chain amino acids [9]. However, the effect of the colonic microbiota and its metabolic profile under high protein on the colonic inflammatory response and barrier function in growing pigs is still not fully understood. The intestinal structure and microbial composition differed among the species (humans, rodents, and pigs) and more investigations are required in pigs.

Soy protein is the main dietary protein for pigs and is considered a high-quality protein derived from soybeans. The role of soy protein in regulating energy metabolism and appetite has received considerable attention[10]. However, there are only a few reports on the effects of soy protein on colonic microbiota and colon health. In this study, soy protein was selected and hydrolysed into soy protein hydrolysate (SPH), which was then injected into a pig's duodenum through a fistula. This model was used in the early stage of our laboratory to explore the effect of SPH on the secretion of intestinal satiety hormones[11]. This model was further used in this study to increase intestinal SPH levels.

We hypothesized that increasing intestinal soy protein levels will change the colonic microbial community and metabolism, thereby affecting colon health in growing pigs. Thus, we utilized the fistula model to explore the effects of SPH infusion on colonic microbiota, colonic inflammation, and barrier function in growing pigs.

\section{Results}

\section{Growth performance and colon index}

Table 2 demonstrates that the average daily feed intake, average daily gain, and ratio of feed to gain were not markedly different between the saline and SPH pigs $(P>0.05)$. Colon index and colon length were lower in the SPH group relative to the saline group $(P<0.05)$.

\section{Effect of SPH on colonic microbiota}

Compared with the saline group, the abundance-based coverage estimator (ACE) and Chao index representing species richness increased in the SPH group $(P<0.05$, Figure $1 \mathrm{~A})$. The Shannon and 
Simpson indexes related to species diversity did not differ between the SPH and saline groups $(P>0.05$, Figure 1B). Moreover, PCoA showed no difference in microbiota composition between the two groups $(P>$ 0.05, Figure 1C).

At the phylum level, Firmicutes and Actinobacteria were the two predominant phyla, contributing $74.36 \%$ and $6.49 \%$ of colonic microbiota in the saline pigs and $75.08 \%$ and $8.25 \%$ in the SPH pigs, respectively. Bacteroidetes and Proteobacteria constituted the next two most dominant phyla at 7.24\% and 0.95\% in saline pigs and $6.37 \%$ and $1.76 \%$ in SPH pigs, respectively (Figure 1D). In addition, SPH treatment increased the abundance of Patescibacteria $(P<0.05$, Figure 1E). At the genus level, Lactobacillus, Ruminococcaceae UCG-014, Lachnospiraceae XPB1014 group, Treponema 2, and Streptococcus were the abundant genera (Figure 1F). Moreover, the abundance of Lachnospiraceae XPB1014 group, Veillonella, Prevotella, Candidatus Saccharimonas, Erysipelotrichaceae UCG-006, Prevotellaceae_uncultured, Escherichia-Shigella, Dialister, and Prevotellaceae UCG-003 was higher, whereas the abundance of the Lachnospiraceae NK4A136 group, Lachnospiraceae_uncultured, Coprococcus 3, Lachnospiraceae UCG-002, and Anaerovibrio was lower in the SPH group relative to the saline group $(P<0.05$, Figure $1 \mathrm{G})$.

Based on the above results, we identified all bacterial OTUs; 1223 OTUs were detected, with 930 OTUs shared between two groups, 136 being unique in the saline pigs and 157 being unique in the SPH pigs (Figure 1H). Among the different OTUs, 10 OTUs underwent transformation corresponding to the genera affected by the SPH. Specifically, in comparison with the saline group $(P<0.05$, Figure $1 \mathrm{I})$, the SPH group showed a lower abundance of OTU87 (g_Coprococcus 3), OTU269 (g_Lachnospiraceae NK4A136 group), and OTU593 (g__Lachnospiraceae UCG-002) and greater abundances of OTU57 (g_Candidatus Saccharimonas), OTU75 (g_Erysipelotrichaceae UCG-006), OTU80 (g_Veillonella), OTU384 (g_Prevotellaceae UCG-003), OTU491 (g_Prevotella), OUT21 (g_Escherichia-Shigella), and OTU338 (g_Dialister) $(P<0.05)$.

\section{Colonic microbial metabolites}

The concentration of SCFAs, biogenic amines, and ammonia was determined as an indicator of microbial fermentation. Compared with the saline pigs, a higher content of isovalerate and lower content of propionate, butyrate, and total SCFAs were observed in the SPH pigs $(P<0.05$, Figure 2A). Moreover, SPH infusion increased the content of cadaverine, putrescine, and total biogenic amines $(P<0.05$, Figure 2B). Furthermore, the ammonia concentration of SPH pigs was higher than that of saline pigs $(P<0.05$, Figure 2C).

Furthermore, we performed Spearman's correlation analysis on different genera and metabolites (Figure 2D). The five genera (Veillonella, Prevotella, Candidatus Saccharimonas, Escherichia-

Shigella, and Dialister) that were markedly increased in abundance from the SPH group were positively correlated with the colonic concentration of ammonia, isovalerate, cadaverine, and putrescine $(P<0.05)$; the five genera that were decreased in abundance from the SPH group, Lachnospiraceae NK4A136 group, Lachnospiraceae_uncultured, Coprococcus 3, and Lachnospiraceae UCG-002 were positively correlated 
with the content of butyrate $(P<0.05)$, and Anaerovibrio was positively correlated with the content of propionate $(P<0.05)$.

\section{Concentration of inflammatory cytokines and expression of tight junction proteins}

Intestinal health in pigs is closely related to microbes and their metabolites. Compared with the saline group, increased levels of TNF-a, IL-1 $\beta$ IIL-6, and IL-8 was found in the colonic mucosa of SPH pigs $(P<$ 0.05 , Figure $3 \mathrm{~A})$. Correlation analysis showed that the concentration of pro-inflammatory cytokines was positively correlated with the abundance of eight different genera and the concentration of ammonia, cadaverine, putrescine, and isovalerate, and negatively correlated with the abundance of five different genera and the content of propionate and butyrate (Figures 2D and 3B). Interestingly, SPH treatment also increased the protein expression of tight junction proteins-such as claudin-1, occluding, and ZO-1-in the colonic mucosa of treated pigs $(P<0.05$, Figure 4$)$.

\section{Discussion}

This study demonstrated that increased intestinal SPH levels could alter colonic microbial metabolism in growing pigs. Interestingly, although SPH treatment promoted the secretion of colonic pro-inflammatory cytokines, it appears not to damage the colonic barrier function. Previous studies have revealed that high protein intake increases the body's sense of fullness, thereby leading to a decrease in feed intake, which may reduce protein intake[12]. Thus, when establishing an animal model of HPD, it is necessary to consider whether there is an increase in the actual protein intake of model animals. In this study, the duodenal infusion of SPH did not alter the average feed intake of pigs, thereby indicating that the model increased the intestinal SPH levels.

An increase in the intestinal protein levels can promote the fermentation of gut microbes. The colon contains a large number of microbes, which are the main players in protein fermentation. Consistent with previous HPD experiments on rats[5], SPH infusion in this study led to an increase in the species richness of colonic microbiota in growing pigs, which may be related to an increase in the abundance of proteinfermenting bacteria and a few harmful bacteria. Moreover, SPH infusion increased the abundance of certain genera. The increases in Erysipelotrichaceae[13], Prevotellaceae_uncultured, Prevotellaceae UCG003[14], and Candidatus Saccharimonas[15] have been shown to be involved in the development of chronic inflammation. A few species within the genera Escherichia-Shigella[16], Dialister[17], Prevotella[18], and Veillonella[19] are regarded as opportunistic pathogens and are relevant to colitis. In addition, Escherichia-Shigella and Veillonella are the main biogenic amine producers in the colon, while Prevotella and Dialister are associated with the production of branched chain fatty acids and ammonia, respectively[16-19]. These results indicate that SPH treatment increased the abundance of bacteria related to protein fermentation and chronic inflammation. On the other hand, there is a reduction in the abundance of SCFAs-producing bacteria and the content of SCFAs in the faeces of people who consume HPD [6]. In this study, the abundance of propionate-producing bacteria Anaerovibrio[20] and a few butyrate-producing bacteria such as Lachnospiraceae NK4A136 group[21], Coprococcus 3[22], 
Lachnospiraceae_uncultured, and Lachnospiraceae UCG-002[23] was markedly decreased in SPH pigs. The decrease in the abundance of these bacterial genera may affect the production of propionate and butyrate, thereby affecting colon health.

The current study further revealed that changes in colonic microbiota were consistent with altered microbial metabolism. SPH treatment reduced the concentration of propionate and butyrate in the colon. A study revealed that athletes who consumed HPD had a reduced concentration of SCFAs in their stool, while athletes who consumed a high-carbohydrate diet had a higher concentration of SCFAs in their stool[24]. Propionate and butyrate have anti-inflammatory effects and can inhibit the expression of adhesion molecules and the production of chemokines[25]. The low concentrations of propionate and butyrate under SPH infusion may also suggest a decrease in immunity. Glutamate and lysine can be fermented by gut microbiota to produce butyrate[26]. The increased content of cadaverine from lysine decarboxylation due to SPH treatment reported in this study suggests an increased transformation of lysine towards cadaverine production rather than butyrate production. Moreover, the concentration of other amino acid metabolites-including putrescine, ammonia, and isovalerate-also significantly increased after SPH infusion. High amounts of putrescine, cadaverine, ammonia, and isovalerate are potentially cytotoxic and can induce oxidative stress and inflammation in the colon[27]. Marakova et al. found that putrescine and cadaverine levels can be used as potential biomarkers for inflammatory bowel disease (IBD)[28]. Compared with healthy individuals, ammonia-intoxicated animals were found to have colitis and colon mucosal histological damage[29]. High concentrations of protein metabolites can cause inflammation and, thus, damage the intestinal barrier function, while physiological concentrations are beneficial to intestinal health. For example, putrescine increases the expression of tight junction proteins, like occludin and ZO-1, by promoting the expression of toll-like receptor 2 in intestinal epithelial cells, thereby maintaining the integrity of the intestinal barrier[30]. In an existing study, piglets intervened with probiotics showed better growth performance and improved physiological structure of the intestine, with a higher content of isovalerate in colonic digesta[31], thereby indicating that isovalerate may be beneficial to the intestinal barrier function. Furthermore, correlation analysis proved that the changes in the production of these metabolites are related to microbiota. Collectively, SPH treatment increased the content of protein-fermented products and reduced the content of SCFAs.

We further evaluated the colonic inflammatory response and barrier function. SPH treatment increased the levels of TNF- $a, I L-1 \beta, I L-6$, and IL-8 in the colonic mucosa of pigs, thereby suggesting that SPH induced colon inflammation. Lya et al. used HPD to feed 35-day-old piglets and found that a HPD caused diarrhoea in piglets by activating intestinal inflammation induced by NF-KB signalling[32]. Colon inflammation may be caused by colonic microbial disorders after SPH intervention, which is consistent with the data of correlation analysis between inflammatory cytokines and different bacteria.

Intestinal inflammation is closely related to intestinal barrier function. Previous studies have revealed that severe colon inflammation can increase intestinal epithelial permeability through the disruption of the tight junction structure[33]. Interestingly, SPH infusion promoted the expression of tight junction proteins -including ZO-1, occluding, and claudin-1-in the colonic mucosa, thereby indicating that increased SPH 
levels may contribute to maintaining colonic barrier integrity. Changes in the intestinal environment using appropriate protein may not impair the colonic barrier function of pigs. Chen et al. evaluated the effects of different dietary protein levels $(12 \%, 15 \%$, and $18 \%$ protein) on the intestinal barrier function of growing pigs and proved that an appropriate increase in dietary protein concentration can promote the expression of tight junction proteins in the colon[34]. High concentrations of protein metabolites are toxic, while physiological concentrations are beneficial to gut health[35]. In this study, appropriate concentrations of biogenic amines and isovalerate were found to cause mild colon inflammation, but these metabolites may also protect the colonic barrier function. Furthermore, mild colonic inflammation and related inflammatory cytokines can promote the repair and strengthening of the barrier[36].

The duodenal fistula model in this study is more suitable for our previous experiments in the small intestine. In the future, it is worth considering that the cecal fistula model is used to directly increase the colonic SPH level. In addition, more indicators related to the evaluation of colon inflammation and barrier function need to be determined in subsequent experiments.

\section{Conclusion}

This study demonstrated that increasing intestinal SPH levels promoted colon inflammation, which was most likely due to the alteration in the composition and metabolism of colonic microbiota using protein. $\mathrm{SPH}$ intervention reduced the abundance of propionate- and butyrate-producing bacteria and the content of propionate and butyrate and increased the abundance of potentially pathogenic bacteria and proteinfermenting bacteria as well as the content of protein-fermented products such as putrescine, cadaverine, ammonia, and isovalerate. Furthermore, SPH intervention might also be conducive to maintaining the colonic barrier function of growing pigs. These findings provide new insights into the effects of soy protein on colon metabolism and health in growing pigs.

\section{Materials And Methods}

\section{SPH preparation}

The SPH was prepared according to a previous method with slight modification[37]. In briefly, soy protein isolate (9010-10-0) (Yuanye Biotechnology, Shanghai, China) was suspended in 10\% (w/v) distilled water, and porcine pepsin (Yuanye Biotechnology, S10027) hydrolysis was conducted at an enzyme-to-substrate ratio of $1: 100(\mathrm{w} / \mathrm{w}), \mathrm{pH} 2.0$, and $37^{\circ} \mathrm{C}$ for $1 \mathrm{~h}$. Thereafter, the $\mathrm{pH}$ of the solution was adjusted to 7.0 with $2 \mathrm{~N} \mathrm{NaOH}$ to terminate the reaction. The obtained hydrolysate was stored at $-20^{\circ} \mathrm{C}$ and lyophilised in a FreeZone 4.5 L Freeze Dry System (Labconco Co., Kansas City, MO, USA) for further use.

\section{Animals and experimental procedures}

Sixteen castrated pigs (Duroc $\times$ Landrace $\times$ Large White, aged 50 days) with an initial weight of $14.5 \pm 0.2$ $\mathrm{kg}$ were obtained from a commercial farm in Jiangsu Province, China. The pigs were kept in separate metabolic cages under a constant temperature of $25 \pm 2^{\circ} \mathrm{C}$ and given unlimited access to water and pig 
feed. After a week of acclimatisation, the pigs were fasted for $12 \mathrm{~h}$ before installing a simple T-cannula in the duodenum[38]. The length, width, and inner diameter of the T-cannula were 8.2, 10.0, and $1.5 \mathrm{~cm}$, respectively. After the surgery, all pigs were hypodermically injected with ceftriaxone sodium and the wound and adjacent skin were disinfected with iodine tincture for one week (twice a day) to avoid potential infection. After they fully recovered from the duodenal fistula surgery over a two-week recuperation period, a two-week short-term experiment of SPH on the secretion of an intestinal satiety hormone was performed[11]. After a week of rest, all pigs were randomly allocated to the saline (control, $n$ $=8)$ group and SPH group $(n=8)$ with no differences in body weight $(35.2 \pm 0.3 \mathrm{~kg})$ and feed intake. The entire experiment was conducted over a period of 16 days, during which the pigs in the saline and SPH groups were infused with $10 \mathrm{ml}$ sterile saline and $10 \mathrm{ml} \mathrm{SPH}$ solution $(70 \mathrm{~g} /$ day $)$, respectively, through a duodenal fistula at 8:00 am and 5:00 pm each day. The SPH solution was adjusted to $\mathrm{pH} 5.0$, which is close to the native $\mathrm{pH}$ of porcine duodenum[39]. The basal diet in the experiment was designed based on the National Research Council (NRC) (2012) (Table 1). The feed consumption of each pig was recorded every day to calculate average feed intake. In addition, the body weights of all pigs were recorded on days 1 and 16 to determine average weight gain.

\section{Sample collection}

After fasting for $12 \mathrm{~h}$ on day 16 , all pigs were given general anaesthesia by an intravenous injection of sodium pentobarbital solution ( $40 \mathrm{mg} / \mathrm{kg}$ body weight), and were sacrificed by jugular exsanguination. The colons of all pigs were taken out within 5 minutes after slaughter and measured for length; fresh digesta from the colon was collected in a sterile tube and immediately stored at $-20^{\circ} \mathrm{C}$ for the analysis of microbiota and metabolites. The colon was weighed after removing all the digesta. In addition, a small piece of the colon tissue was collected and washed with phosphate buffered solution (PBS, pH 7.0); then, the colonic mucosa was scraped and immediately frozen in liquid nitrogen, and used to determine the concentration of inflammatory cytokines and the protein expression of tight junction proteins.

\section{Analysis of colonic microbial metabolites}

The content of short-chain fatty acids (SCFAs) was determined using the method of Wang et al. [40]. Briefly, $1.5 \mathrm{ml}$ of double distilled water was added into $0.4 \mathrm{~g}$ of digesta. The mixture was mixed and centrifuged $\left(13,400 \times \mathrm{g}, 4^{\circ} \mathrm{C}, 10 \mathrm{~min}\right)$ in order to obtain supernatants. $1 \mathrm{ml}$ supernatant and $200 \mu \mathrm{L}$ of $25 \%(\mathrm{w} / \mathrm{v})$ metaphosphoric acid were mixed and kept overnight at $-20^{\circ} \mathrm{C}$. Then, the supernatant was centrifuged $\left(13,400 \times \mathrm{g}, 4^{\circ} \mathrm{C}, 10 \mathrm{~min}\right)$ and filtered with a $0.22 \mu \mathrm{L}$ filter, and then measured with an Agilent 7890B gas chromatograph.

High-performance liquid chromatography (HPLC) was selected to detect the content of biogenic amines according to the method given by Yang et al.[41]. For this purpose, $0.6 \mathrm{~g}$ of digesta was weighed and 1.5 $\mathrm{ml}$ of trichloroacetic acid solution was added to precipitate the proteins and peptides. The sample was extracted with n-hexane and derived with dansyl chloride. Gradient elution of two solvents was carried out in the following manner: solvent A and solvent B were HPLC grade water and acetonitrile respectively, and the flow rate was set to $1.0 \mathrm{~mL} / \mathrm{min}$. 
In addition, $0.1 \mathrm{~g}$ of digesta was prepared and acidified with $0.9 \mathrm{ml}$ of $0.2 \mathrm{~mol} / \mathrm{L} \mathrm{HCl}$ for the determination of ammonia content using a spectrophotometer (UV-2450; Shimadzu, Tokyo, Japan) following the previous method[42].

\section{S rRNA sequencing}

DNA from different colonic digesta was extracted using a DNA extraction kit (Qiagen, Hilden, Germany) following the manufacturer's instructions. DNA concentration and purity were measured. The primers $515 \mathrm{~F}$ and $907 \mathrm{R}$ were used to amplify the V4-V5 region of the $16 \mathrm{~S}$ rRNA gene according to the previous methods[43]. Thereafter, the amplicon libraries were established using NEB Next ${ }^{\circ} U$ ltra ${ }^{T M}$ DNA Library Prep Kit for Illumina (NEB, USA) based on the manufacturer's illustrations. Then, the library was sequenced on an Illumina MiSeq platform. QIIME (version 1.70) was used to demultiplex and quality-filter the raw FASTQ files. Sequences with $\geq 97 \%$ similarity were classified as one operational taxonomic unit (OTU). The representative sequence of each OTU was selected, and the Ribosomal Database Project (RDP) classifier was used to annotate taxonomic information. The OTU table was refined to calculate alpha diversity. Beta diversity was calculated based on principal coordinates analysis (PCOA) and Unweighted Pair Group Method with Arithmetic Mean (UPGMA). Sequence alignment was performed with BLAST, and the feature sequences were annotated for each representative sequence using the SILVA database (Release 132) to determine the different taxonomies.

\section{Cytokine assay}

The levels of interleukin (IL)-6, IL-1 $\beta$, IL-8, IL-10, and tumour necrosis factor- $a$ (TNF-a) in the colon mucosa supernatants were quantified using ELISA kits (Nanjing Jiancheng Bioengineering Institution, Nanjing, China) under manufacturer's recommendations.

\section{Western blotting assay}

The expression of tight junction proteins of the colon was determined by western blotting analysis based on the previous method[44]. The primary antibodies used include anti-zonula occludens 1 (ZO-1) (1:2,000, Abcam, ab211737), anti-occludin (1:1,000, Abcam, ab167161), anti-claudin-1 (1:1,000, CST, \#5406), and anti- $\beta$-actin (1:1,000, Abcam, ab8226). Horseradish peroxidase-conjugated AffiniPure goat anti-mouse IgG $(H+L)(1: 5,000$, Thermo Pierce, 31160) or goat anti-rabbit IgG $(H+L)(1: 5,000$, Jackson, Thermo Pierce, 31210) were used as the secondary antibody. Image $\mathrm{J}$ software ( $\mathrm{NIH}$, Bethesda, MD, USA) was used to perform densitometry analysis.

\section{Statistical analysis}

Data pertaining to the growth performance, intestinal index, microbial metabolites, protein expression of cytokines, and tight junction proteins were presented as mean \pm standard error of mean (SEM). All data were compared in SPSS 20.0 (SPSS Inc., Chicago, USA) and graphs were drawn using GraphPad Prism 8.0.2 (La Jolla, CA, USA). Furthermore, the Student's t-test was performed to analyze the difference significance, and significant differences were deemed when $P<0.05$. 
The alpha diversity was examined by the Student's t-test. Different bacteria were compared by the MannWhitney $U$ test with multiple comparisons adjusted by the Benjamini-Hochberg false discovery rate (FDR). The correlation among the abundance of different genera, the concentration of microbial metabolites, and mucosal inflammatory cytokines were analyzed according to Spearman's correlation analysis (XLStat software; Addinsoft).

\section{Abbreviations}

HPD: high-protein diets; SPH: soy protein hydrolysate; SCFAs: short-chain fatty acids; HPLC: highperformance liquid chromatography; OTUs: operational taxonomic units; RDP: Ribosomal Database Project; PCoA: principal coordinates analysis; UPGMA: Unweighted Pair Group Method with Arithmetic Mean; IL: interleukin; TNF-a: tumour necrosis factor-a; ZO-1: zonula occludens 1; FDR: false discovery rate; ACE: abundance-based coverage estimator; IBD: inflammatory bowel disease.

\section{Declarations}

\section{Acknowledgements}

We thank Jangyin Feng, Zhen Zhang, and Lvyang Wang for their assistance in sample collection.

\section{Author contributions}

S.H. and Z.L. conceived and designed the experiments. Z.L. and L.D. performed the experiments. Z.L. and $\mathrm{SH}$ analysed the data and drafted the manuscript. S.H. and W.Z. provided the funding.

\section{Funding}

This research was funded by the National Key Basic Research Program of China (2013CB127301).

\section{Availability of data and materials}

The 16S rRNA gene sequencing data on which the conclusions of the manuscript rely have been deposited in the National Center for Biotechnology Information (NCBI) database (accession number: SRP347652).

\section{Ethics approval}

The experimental design and procedures of the current study were reviewed and approved by the Experimental Animal Welfare Ethics Committee of Nanjing Agricultural University (approval number SYXK-2017-0027), and ARRIVE guidelines and guidelines for animal welfare of the Chinese were followed.

\section{Consent for publication}


Not applicable.

\section{Conflicts of interest}

There are no conflicts of interest to declare that may inappropriately influence our work.

\section{References}

1. Gao J, Yin J, Xu K, Li T, Yin Y. What Is the Impact of Diet on Nutritional Diarrhea Associated with Gut Microbiota in Weaning Piglets: A System Review. BIOMED RES INT. 2019;2019:6916189.

2. Wu Y, Jiang Z, Zheng C, Wang L, Zhu C, Yang X, et al. Effects of protein sources and levels in antibiotic-free diets on diarrhea, intestinal morphology, and expression of tight junctions in weaned piglets. Anim Nutr. 2015;1:170-176.

3. Zhang H, Wielen NV, Hee BV, Wang J, Hendriks W, Gilbert M. Impact of Fermentable Protein, by Feeding High Protein Diets, on Microbial Composition, Microbial Catabolic Activity, Gut Health and beyond in Pigs. Microorganisms. 2020;8.

4. Fan P, Li L, Rezaei A, Eslamfam S, Che D, Ma X. Metabolites of Dietary Protein and Peptides by Intestinal Microbes and their Impacts on Gut. Curr Protein Pept Sci. 2015;16:646-654.

5. Mu C, Yang Y, Luo Z, Guan L, Zhu W. The Colonic Microbiome and Epithelial Transcriptome Are Altered in Rats Fed a High-Protein Diet Compared with a Normal-Protein Diet. J NUTR. 2016;146:474-483.

6. Russell WR, Gratz SW, Duncan SH, Holtrop G, Ince J, Scobbie L, et al. High-protein, reducedcarbohydrate weight-loss diets promote metabolite profiles likely to be detrimental to colonic health. AM J CLIN NUTR. 2011;93:1062-1072.

7. Richter JF, Pieper R, Zakrzewski SS, Gunzel D, Schulzke JD, Van Kessel AG. Diets high in fermentable protein and fibre alter tight junction protein composition with minor effects on barrier function in piglet colon. Br J Nutr. 2014;111:1040-1049.

8. Zhou L, Fang L, Sun Y, Su Y, Zhu W. Effects of the dietary protein level on the microbial composition and metabolomic profile in the hindgut of the pig. ANAEROBE. 2016;38:61-69.

9. Cone JW, Jongbloed AW, Van Gelder AH, de Lange L. Estimation of protein fermentation in the large intestine of pigs using a gas production technique. ANIM FEED SCI TECH. 2005;123:463-472.

10. Velasquez MT, Bhathena SJ. Role of dietary soy protein in obesity. INT J MED SCI. 2007;4:72-82.

11. Wang L, Ding L, Zhu W, Hang S. Soybean protein hydrolysate stimulated cholecystokinin secretion and inhibited feed intake through calcium-sensing receptors and intracellular calcium signalling in pigs. FOOD FUNCT. 2021;12:9286-9299.

12. Kohanmoo A, Faghih S, Akhlaghi M. Effect of short- and long-term protein consumption on appetite and appetite-regulating gastrointestinal hormones, a systematic review and meta-analysis of randomized controlled trials. PHYSIOL BEHAV. 2020;226. 
13. Dinh DM, Volpe GE, Duffalo C, Bhalchandra S, Tai AK, Kane AV, et al. Intestinal microbiota, microbial translocation, and systemic inflammation in chronic HIV infection. J INFECT DIS. 2015;211:19-27.

14. Sun L, Jia H, Li J, Yu M, Yang Y, Tian D, et al. Cecal Gut Microbiota and Metabolites Might Contribute to the Severity of Acute Myocardial Ischemia by Impacting the Intestinal Permeability, Oxidative Stress, and Energy Metabolism. FRONT MICROBIOL. 2019;10.

15. Camelo-Castillo AJ, Mira A, Pico A, Nibali L, Henderson B, Donos N, et al. Subgingival microbiota in health compared to periodontitis and the influence of smoking. FRONT MICROBIOL. 2015;6:119.

16. Di Martino ML, Campilongo R, Casalino M, Micheli G, Colonna B, Prosseda G. Polyamines: Emerging players in bacteria-host interactions. INT J MED MICROBIOL. 2013;303:484-491.

17. Yang Y, Wu H, Dong S, Jin W, Han K, Ren Y, et al. Glycation of fish protein impacts its fermentation metabolites and gut microbiota during in vitro human colonic fermentation. FOOD RES INT. 2018;113:189-196.

18. Larsen JM. The immune response to Prevotella bacteria in chronic inflammatory disease. IMMUNOLOGY. 2017;151:363-374.

19. De Cruz P, Kang S, Wagner J, Buckley M, Sim WH, Prideaux L, et al. Association between specific mucosa-associated microbiota in Crohn's disease at the time of resection and subsequent disease recurrence: A pilot study. J GASTROEN HEPATOL. 2015;30:268-278.

20. Prins RA, Lankhorst A, van der Meer P, Van Nevel CJ. Some characteristics of Anaerovibrio lipolytica a rumen lipolytic organism. Antonie Van Leeuwenhoek. 1975;41:1-11.

21. Ma L, Ni Y, Wang Z, Tu W, Ni L, Zhuge F, et al. Spermidine improves gut barrier integrity and gut microbiota function in diet-induced obese mice. GUT MICROBES. 2020;12.

22. Ma T, Villot C, Renaud D, Skidmore A, Chevaux E, Steele M, et al. Linking perturbations to temporal changes in diversity, stability, and compositions of neonatal calf gut microbiota: prediction of diarrhea. ISME J. 2020;14:2223-2235.

23. Mancabelli L, Milani C, Lugli GA, Turroni F, Mangifesta M, Viappiani A, et al. Unveiling the gut microbiota composition and functionality associated with constipation through metagenomic analyses. Sci Rep. 2017;7:9879.

24. Son J, Jang LG, Kim BY, Lee S, Park H. The Effect of Athletes' Probiotic Intake May Depend on Protein and Dietary Fiber Intake. NUTRIENTS. 2020;12.

25. Vinolo MAR, Rodrigues HG, Nachbar RT, Curi R. Regulation of Inflammation by Short Chain Fatty Acids. NUTRIENTS. 2011;3:858-876.

26. Louis P, Flint HJ. Formation of propionate and butyrate by the human colonic microbiota. ENVIRON MICROBIOL. 2017;19:29-41.

27. Di Martino ML, Campilongo R, Casalino M, Micheli G, Colonna B, Prosseda G. Polyamines: Emerging players in bacteria-host interactions. INT J MED MICROBIOL. 2013;303:484-491.

28. Marakova K, Piestansky J, Zelinkova Z, Mikus P. Simultaneous determination of twelve biogenic amines in human urine as potential biomarkers of inflammatory bowel diseases by capillary 
electrophoresis - tandem mass spectrometry. J Pharm Biomed Anal. 2020;186:113294.

29. Visek WJ. Diet and cell growth modulation by ammonia. AM J CLIN NUTR. 1978;31:S216-S220.

30. Chen J, Rao JN, Zou T, Liu L, Marasa BS, Xiao L, et al. Polyamines are required for expression of Tolllike receptor 2 modulating intestinal epithelial barrier integrity. AM J PHYSIOL-GASTR L. 2007;293:G568-G576.

31. Liu H, Zhang J, Zhang S, Yang F, Thacker PA, Zhang G, et al. Oral administration of Lactobacillus fermentum 15007 favors intestinal development and alters the intestinal microbiota in formula-fed piglets. J Agric Food Chem. 2014;62:860-866.

32. Lya B, Jla C, Mw A, Qw A, JI A, Nd C, et al. Dietary high protein-induced diarrhea and intestinal inflammation by activation of NF-KB signaling in piglets. Animal Nutrition. 2021.

33. Abreu MT, Palladino AA, Arnold ET, Kwon RS, McRoberts JA. Modulation of barrier function during Fas-mediated apoptosis in human intestinal epithelial cells. GASTROENTEROLOGY. 2000;119:15241536.

34. Chen X, Song P, Fan P, He T, Jacobs D, Levesque CL, et al. Moderate Dietary Protein Restriction Optimized Gut Microbiota and Mucosal Barrier in Growing Pig Model. Front Cell Infect Microbiol. 2018;8:246.

35. Windey K, De Preter V, Verbeke K. Relevance of protein fermentation to gut health. MOL NUTR FOOD RES. 2012;56:184-196.

36. Luissint AC, Parkos CA, Nusrat A. Inflammation and the Intestinal Barrier: Leukocyte-Epithelial Cell Interactions, Cell Junction Remodeling, and Mucosal Repair. GASTROENTEROLOGY. 2016;151:616632.

37. Kim SS, Ahn C, Moon SW, Je J. Purification and antioxidant activities of peptides from sea squirt (Halocynthia roretzi) protein hydrolysates using pepsin hydrolysis. FOOD BIOSCI. 2018;25:128-133.

38. Jongbloed AW, Mroz Z, Kemme PA. The effect of supplementary Aspergillus niger phytase in diets for pigs on concentration and apparent digestibility of dry matter, total phosphorus, and phytic acid in different sections of the alimentary tract. J ANIM SCI. 1992;70:1159-1168.

39. Abello J, Corring T, Laplace JP. Contribution of bile and pancreatic juice to the control of pH in the pig duodenum. Reprod Nutr Dev. 1987;27:681-687.

40. Wang XF, Mao SY, Liu JH, Zhang LL, Cheng YF, Jin W, et al. Effect of the gynosaponin on methane production and microbe numbers in a fungus-methanogen co-culture. J ANIM FEED SCI. 2011;20:272-284.

41. Zhang Y, Yu K, Chen H, Su Y, Zhu W. Caecal infusion of the short-chain fatty acid propionate affects the microbiota and expression of inflammatory cytokines in the colon in a fistula pig model. MICROB BIOTECHNOL. 2018;11:859-868.

42. Nyachoti CM, Omogbenigun FO, Rademacher M, Blank G. Performance responses and indicators of gastrointestinal health in early-weaned pigs fed low-protein amino acid-supplemented diets. J ANIM SCI. 2006;84:125-134. 
43. Sun Y, Zhou L, Fang L, Su Y, Zhu W. Responses in colonic microbial community and gene expression of pigs to a long-term high resistant starch diet. FRONT MICROBIOL. 2015;6:877.

44. Meng Y, Zhang J, Zhang F, Ai W, Zhu X, Shu G, et al. Lauric Acid Stimulates Mammary Gland Development of Pubertal Mice through Activation of GPR84 and PI3K/Akt Signaling Pathway. J Agric Food Chem. 2017;65:95-103.

\section{Tables}


Table 1

Dietary composition and nutritional component (air dry basis).

\begin{tabular}{|c|c|}
\hline Items & Composition, \% \\
\hline \multicolumn{2}{|l|}{ Ingredients } \\
\hline Corn & 59.81 \\
\hline Soya bean meal & 25.80 \\
\hline Wheat flour & 5.00 \\
\hline Rice bran meal & 3.00 \\
\hline Corn germ meal & 3.00 \\
\hline L-Lysine, 98.5\% & 0.11 \\
\hline DL-Methionine, 99.0\% & 0.04 \\
\hline L-Threonine, 99.0\% & 0.04 \\
\hline L-Tryptophan, 99.0\% & 0.01 \\
\hline Calcium hydrogen phosphate & 0.40 \\
\hline Limestone & 1.00 \\
\hline $\mathrm{NaCl}$ & 0.50 \\
\hline Acidifying agent & 0.40 \\
\hline Zinc oxide & 0.20 \\
\hline Choline chloride & 0.10 \\
\hline Mould inhibitor & 0.05 \\
\hline Phytase & 0.02 \\
\hline Compound enzyme & 0.02 \\
\hline Mineral premix ${ }^{a}$ & 0.50 \\
\hline \multicolumn{2}{|l|}{ Nutrition level, \% } \\
\hline $\mathrm{NE}, \mathrm{MJ} / \mathrm{Kg}$ & 10.19 \\
\hline Crude protein & 18.00 \\
\hline
\end{tabular}

a Premix supplied the following per kg complete diet: vitamin $A, 3,800 \mathrm{IU}$; vitamin $\mathrm{D}_{3}, 800 \mathrm{IU}$; vitamin $\mathrm{E}$, 9 mg; vitamin $B_{1}, 1$ mg; vitamin $\mathrm{K}_{3}, 1$ mg; vitamin $B_{2}, 2$ mg; vitamin $B_{6}, 1.2$ mg; vitamin $B_{12}, 10 \mu \mathrm{g}$; nicotinic acid, $10 \mathrm{mg}$; biotin, $50 \mu \mathrm{g}$; folic acid, $0.4 \mathrm{mg}$; iron (as $\mathrm{FeSO}_{4} \cdot \mathrm{H}_{2} \mathrm{O}$ ), $80 \mathrm{mg}$; zinc, $80 \mathrm{mg}$; iodine (as KI), $0.14 \mathrm{mg}$; $\mathrm{Se}\left(\right.$ as $\left.\mathrm{Na}_{2} \mathrm{SeO}_{3}\right), 0.25 \mathrm{mg}$; copper as $\left(\mathrm{CuSO}_{4} \cdot 5 \mathrm{H}_{2} \mathrm{O}\right), 5 \mathrm{mg}$; and $\mathrm{Mn}\left(\right.$ as $\mathrm{MnSO}_{4}$. $\left.\mathrm{H}_{2} \mathrm{O}\right), 3 \mathrm{mg}$. 


\begin{tabular}{|c|c|}
\hline Items & Composition, \% \\
\hline Crude fat & 2.63 \\
\hline Crude fiber & 3.79 \\
\hline Crude ash & 5.03 \\
\hline Lysine & 1.10 \\
\hline Methionine & 0.34 \\
\hline Tryptophan & 0.21 \\
\hline Threonine & 0.75 \\
\hline Valine & 0.87 \\
\hline \multicolumn{2}{|c|}{$\begin{array}{l}\text { a Premix supplied the following per kg complete diet: vitamin } \mathrm{A}, 3,800 \mathrm{lU} \text {; vitamin } \mathrm{D}_{3}, 800 \mathrm{IU} \text {; vitamin } \mathrm{E} \\
9 \mathrm{mg} \text {; vitamin } \mathrm{B}_{1}, 1 \mathrm{mg} \text {; vitamin } \mathrm{K}_{3}, 1 \mathrm{mg} \text {; vitamin } \mathrm{B}_{2}, 2 \mathrm{mg} \text {; vitamin } \mathrm{B}_{6}, 1.2 \mathrm{mg} \text {; vitamin } \mathrm{B}_{12}, 10 \mu \mathrm{\mu g} \text {; } \\
\text { nicotinic acid, } 10 \mathrm{mg} \text {; biotin, } 50 \mu \mathrm{g} \text {; folic acid, } 0.4 \mathrm{mg} \text {; iron }\left(\text { as } \mathrm{FeSO}_{4} \cdot \mathrm{H}_{2} \mathrm{O}\right), 80 \mathrm{mg} \text {; zinc, } 80 \mathrm{mg} \text {; iodine } \\
(\text { as } \mathrm{KI}), 0.14 \mathrm{mg} \text {; } \mathrm{Se}\left(\text { as } \mathrm{Na}_{2} \mathrm{SeO}_{3}\right), 0.25 \mathrm{mg} \text {; copper as }\left(\mathrm{CuSO}_{4} \cdot 5 \mathrm{H}_{2} \mathrm{O}\right), 5 \mathrm{mg} \text {; and } \mathrm{Mn}\left(\text { as } \mathrm{MnSO}_{4} \text {. }\right. \\
\left.\mathrm{H}_{2} \mathrm{O}\right), 3 \mathrm{mg} \text {. }\end{array}$} \\
\hline
\end{tabular}

Table 2

Growth performance and colon index in the saline and SPH groups.

\begin{tabular}{|llll|}
\hline Items & Saline & SPH & $P$-value \\
\hline Average daily feed intake $(\mathrm{kg} / \mathrm{d})$ & $1.73 \pm 0.07$ & $1.61 \pm 0.07$ & 0.236 \\
\hline Average daily gain $(\mathrm{kg})$ & $0.87 \pm 0.04$ & $0.75 \pm 0.06$ & 0.095 \\
\hline Feed conversion ratio & $2.06 \pm 0.10$ & $2.13 \pm 0.18$ & 0.737 \\
\hline Colon index $(\%)$ & $4.57 \pm 0.08$ & $3.77 \pm 0.29$ & 0.020 \\
\hline Colon length $(\mathrm{m})$ & $3.71 \pm 0.12$ & $3.27 \pm 0.11$ & 0.016 \\
\hline $\begin{array}{l}\text { Values are mean } \pm \mathrm{SEM}(\mathrm{n}=8) . P<0.05 \text { implies statistically significant. Colon index }=\text { (colon } \\
\text { weight/body weight) } \times 100 \% \text {. }\end{array}$ & & \\
\hline
\end{tabular}

\section{Figures}

\section{Figure 1}

Changes in the microbial composition of colonic digesta with SPH treatment $(n=8)$. (A) The abundancebased coverage estimator (ACE), Chao index, and (B) the Simpson and Shannon index of colonic microbiota. (C) Principal coordinates analysis plots (PCOA) of colonic microbiota. (D) Relative abundance of the microbial phylum and (E) different phyla (Mann-Whitney U-test; median values). (F) Relative 
abundance of the microbial genus and (G) different genera (Mann-Whitney U-test; median values). (H) Analysis of the Venn diagram reveals the total number of OTUs both shared and unique. (I) Significantly different OTUs bubble chart. * $P<0.05$.

\section{Figure 2}

Colonic microbial metabolites and correlation analysis $(n=8)$. (A) Concentrations of SCFAs, (B) biogenic amines, and (C) ammonia in the colonic digesta of saline and SPH pigs. Values are mean \pm SEM. (D) Spearman's correlation analysis of different genera with metabolites and inflammatory cytokines. ${ }^{*} \mathrm{P}<$ 0.05 and $* * P<0.01$.

A

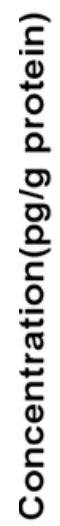

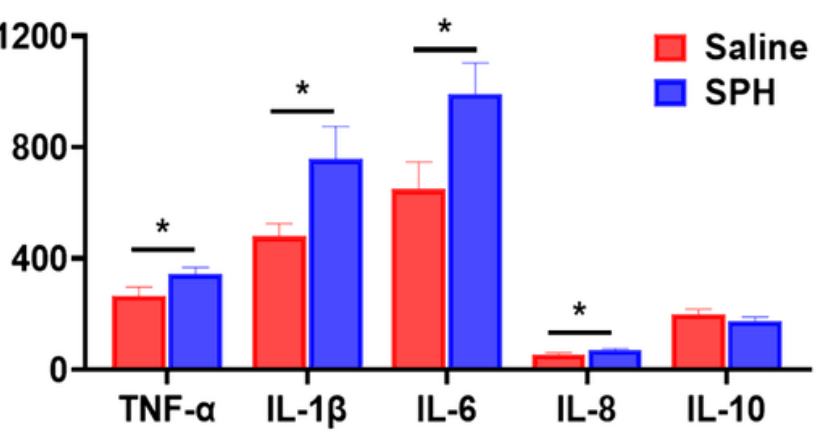

B

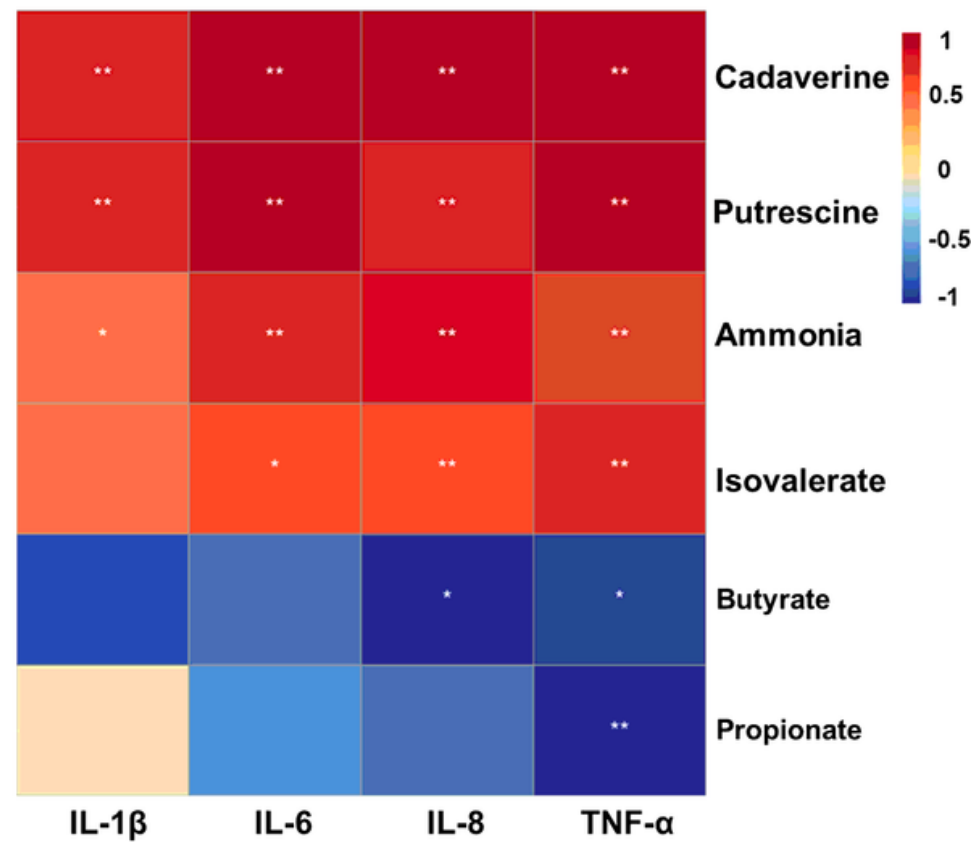

\section{Figure 3}

Inflammatory cytokines and correlation analysis $(n=8)$. (A) Concentrations of inflammatory cytokines in colonic mucosa in saline and SPH groups. Values are mean \pm SEM. (B) Spearman's correlation analysis between the concentration of metabolites and the concentration of inflammatory cytokines. ${ }^{*} P<0.05$ and $* * \mathrm{P}<0.01$.

\section{Figure 4}

The expression of claudin-1, occlusion, and ZO-1 in colonic mucosa. Values are mean \pm SEM $(n=3) .{ }^{*}<<$ 0.05 . 